\title{
Arte Como Opción Antagonista a la Violencia en Población Penal Juvenil de Costa
}

\section{Rica}

\section{Hilda Rodríguez Alfaro \\ Universidad de Iberoamerica}

\section{Resumen:}

A manera de Trabajo Comunal Universitario se lleva a cabo un taller artístico en el Centro Especializado Adulto Joven del Programa Nacional de Atención a la Población Penal Juvenil de la Dirección General de Adaptación Social del Ministerio de Justicia y Paz ubicado en La Reforma, San Rafael de Alajuela. Se titula: Taller Artístico en Cuatro Áreas y tiene como objetivo proponer el arte como opción de expresión sana de sentimientos, emociones y vivencias para los privados de libertad que oscilan entre los 18 y los 25 años de edad. Se pretende introducir el aprendizaje de la Música, Las Artes Plásticas, la Danza y las Artes Dramáticas por un periodo de cuatro meses durante los cuales la actividad central se especificó en la creación, práctica y presentación de coreografías en Hip Hop y baile Folclórico por parte del grupo coreográfico creado dentro de la institución denominado “Danza Libre” a cargo de María Fernanda Mora Clavo, máster en Trabajo Social actual empleada de la institución. Se logra determinar la importancia de opciones saludables como el arte para una adecuada expresión emocional-psicológica de los privados de libertad en contraposición a expresiones por medio de la violencia.

Palabras clave: Arte, expresión artística, privado de libertad, población penal juvenil.

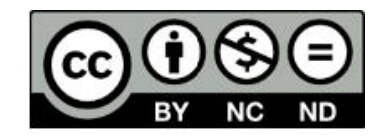




\section{Introducción}

El Programa Nacional de Atención a la Población Penal Juvenil es la instancia de la Dirección General de Adaptación Social, del Ministerio de Justicia y Paz, responsable de la atención de la población que, siendo menor de edad, ha cometido algún tipo de delito. Se atiende a adolescentes y jóvenes de ambos sexos, que son remitidos por las autoridades judiciales del país.

El programa está conformado por:

\section{Dirección del Programa}

2. Centro de Formación Juvenil Zurquí

\section{Centro Especializado Adulto Joven}

4. Centro de Oportunidades Juveniles

5. Programa de Sanciones Alternativas

Antes del año de 1996, era la Ley de Jurisdicción Tutelar de Menores del 21 de diciembre del año 1963, la que regía esta población. Históricamente se contaba en Costa Rica con dos centros de atención para población penal juvenil: uno para niñas y adolescentes, conocido como Centro de Orientación Juvenil Amparo de Zeledón y otro para la población menor de edad masculina llamado Reformatorio San Dimas, ubicado desde el año de 1936 hasta el año de 1973 en Barrio Quesada Durán, instalaciones que hoy día las ocupa el Liceo

\section{Pyes.o.


Castro Madriz y que luego se le denominó Centro de Orientación Juvenil Luis Felipe González Flores, ubicado en Tierra Blanca de Cartago, hasta el año de 1981, en que se reubica a una nueva edificación en San Rafael de Ojo de Agua, Alajuela, que hoy es el CAI San Rafael, con población adulta (Mora y Gámez, 2001).

Las autoridades penitenciarias acondicionaron un espacio físico al interior del CAI La Reforma, adscrito al Nivel Institucional de Adultos que se conocía como Puesto 9, mismo que se hizo insuficiente, por el aumento de la población, lo que provocó que los jóvenes fuesen reubicados en el año de 1997, en otra zona conocida como "Casona”, espacio que hoy día pertenece al CAI San Rafael. Fue el 25 de abril del 2003, por una resolución de todos los Jueces de Ejecución de la Pena, que se ordena entre otras cosas que:

"La Unidad Adulto Joven será inmediatamente adscrita al Programa Nacional de Atención a la Población Penal Juvenil (de ahora en adelante Programa). Su personal técnico, administrativo y de seguridad deberá ser capacitado en materia penal juvenil.

La Coordinadora del Programa supervisará el Proyecto de centro cumpliendo con las funciones establecidas en el artículo 52 del Reglamento Orgánico y Operativo de la Dirección General de Adaptación Social...” (Mora y Gámez, 2001).

A partir de esta fecha, el Centro Especializado Adulto Joven, es un componente más del Programa Nacional de Atención Penal Juvenil, pero continúa dependiendo del CAI La Reforma en algunos aspectos de orden administrativo y de Seguridad. Cuenta con una infraestructura independiente, ubicado en zona aledaña al Centro de Atención Institucional (CAI) La Reforma, que fue inaugurada el 2 de septiembre del 2005 (Mora y Gámez, 2001). 
En el mes de enero del año 2010 el Centro Especializado Adulto Joven fue cerrado por orden judicial debido a que enfrentaba una sobrepoblación y hacinamiento significativos. A falta de otras instalaciones la población adulta joven continuó ingresando al Centro Juvenil Zurquí generando un problema ya que en ese Centro se atendía población menor de edad. El Ministerio de Justicia quedó comprometido a la construcción de nuevas instalaciones para albergar a la población adulta joven en el menor tiempo posible. No obstante fue hasta el 2015, cinco años después, que esas instalaciones están listas para estrenarse en la misma área geográfica del Centro Adulto Joven en la Reforma. Se construyó un módulo para 68 jóvenes privados de libertad, un módulo de atención individualizada que tiene capacidad para 6 privados de libertad, cuatro dormitorios de visita conyugal, cuatro locutorios de atención además de otros edificios administrativos.

Estas instalaciones permitieron trasladar a 68 de los 110 jóvenes privados de libertad que se encontraban en el Centro Juvenil Zurquí adonde pertenecían según su situación jurídica y condición etaria. Sin embargo, es importante aclarar que las instalaciones nuevas del Centro Adulto Joven han sido habitadas no solo por los jóvenes que vienen del Zurquí sino que hay un grupo del Centro Adulto Joven que también ha sido reubicado en esas instalaciones. De esta manera se concluye que de los 68 jóvenes provenientes del Zurquí, aproximadamente 28 se ubicarán en las secciones actuales del Centro Adulto Joven, mismas que implican mayor contención y por ende la población que allí se ubica se caracteriza por una problemática mayor en su convivencia. Lo anterior también tiene que ver con los perfiles que se definieron para los módulos nuevos, denominados Sección E, en los cuales se reubicó 
un estimado de 40 jóvenes del Centro Juvenil Zurquí y un aproximado de 28 jóvenes del Centro Adulto Joven para un total de 68 jóvenes. Estas listas fueron trabajadas por los Equipos Técnicos de cada centro y pretenden responder a un perfil de buena convivencia para un espacio que fue diseñado para población de baja contención.

Sin embargo, el proceso de reubicación ha generado un clima de mucha tensión en la población penal juvenil. Para comprender esta dimensión es importante mencionar que para los jóvenes privados de libertad es fundamental la certeza de su espacio físico en función de la protección de su integridad personal. Las alianzas internas y externas, las luchas de poder, liderazgos y otros, son circunstancias presentes en la condición de encierro que caracteriza la dinámica convivencial en todas las secciones y módulos. En este contexto, una reubicación a un nuevo espacio con otros compañeros, líderes y formas de interrelación resulta amenazante para cualquier joven que sea candidato a este movimiento.

Desde este trabajo comunal universitario se pretende abordar a un grupo representativo de la situación de reubicación para realizar una exploración de sentimientos relacionados con la experiencia de enfrentar este cambio. Temores, fantasías, amenazas reales y subjetivas y cualquier otra vivencia emocional que les despierte el nuevo escenario convivencial. Se plantea realizar esta exploración de sentimientos a través de diferentes formas de expresiones artísticas considerando la importancia de la expresión de sentimientos a través del arte como óptima y de gran enriquecimiento en una población que está sumamente limitada en la expresión verbal o en la apertura de canales de comunicación por muchas y diferentes razones que van desde la edad, la madurez, la etapa restrictiva por la 
que atraviesan hasta las incontables privaciones socioculturales y manejo de códigos verbales muy propios de pandillas o grupos a los que pertenecen.

El taller propone entonces llevar a cabo una intervención artística como método efectivo para la expresión psicológica-emocional de la población privada de libertad y desestimar que se busquen opciones no favorables como la violencia para transmitir sentimientos. Se trata de fortalecer el arte como una opción alterna a la hora de canalizar vivencias sobre todo en el marco de la experiencia de reubicación en un nuevo espacio carcelario, así como colaborar con el personal técnico profesional del Centro Adulto Joven, aportando aspectos que sustenten y enriquezcan la atención técnica en el tema específico de la convivencia en la nueva sección. Elementos como las filiaciones, luchas de poder, liderazgos, relaciones interpersonales y diferentes vivencias que los jóvenes estén enfrentado en su nueva experiencia convivencial, coadyuvan en los objetivos del abordaje que se hace desde el equipo de profesionales del Centro. Además el proyecto apunta a colaborar con los procesos de una sana reinserción social, de contacto interpersonal, de trabajo de grupo, de recreación integral entre otros aspectos que se rescatan de una experiencia de iniciación y contacto con el arte.

\section{Objetivo General:}

Aplicar valores artísticos como métodos de expresión de pensamientos y sentimientos antagonistas de la violencia.

\section{Objetivos Específicos:}


1. Iniciar a los participantes en una vivencia artística.

2. Fomentar la utilización sana del tiempo libre por medio del arte.

3. Desarrollar en los participantes respeto y validación hacia sus propias ideas y hacia las de los demás a pesar de las diferencias.

4. Definir capacidades de logro y de cumplimiento de metas por medio de la realización y finalización de proyectos artísticos.

\section{Método}

Durante 49 sesiones de aproximadamente 4 horas cada una, se trabaja con población privada de liberta del Centro Especializado Adulto Joven en la introducción a cuatro áreas artísticas: Música, Artes Dramáticas, Danza y Artes Plásticas. De las cuatro propuestas artísticas, dos fueron mejor recibidas (Danza y Artes Plásticas) por cuestiones del uso de las instalaciones y disposición en cantidad y tiempo del personal de seguridad que debe acompañar cada una de las sesiones artísticas, por lo que se extiende y profundiza el trabajo en estas dos áreas.

Participaron un total de 37 muchachos de entre los 18 y los 25 años de edad provenientes de las siguientes secciones penitenciarias de este Centro: 8 jóvenes de la sección 
B2, 12 jóvenes de la sección C2, 5 jóvenes de la sección $\mathrm{C} 1,8$ jóvenes de la sección $\mathrm{E}, 3$ jóvenes de la sección D1 y 1 miembro de la sección de Máxima Seguridad.

Las primeras cuatro secciones mencionadas conforman el grupo coreográfico "Danza Libre" quien durante el desarrollo del taller artístico preparó y presentó tres coreografías: Una en Hip Hop, una en estilo Dance Hall y una en Danza Folclórica. Los jóvenes responden mejor a la música de su interés y de sus gustos, por lo que únicamente tres privados de libertad participaron en la Danza Folclórica.

Los jóvenes son convocados a estar listos a una hora determinada para la clase y ensayo de coreografías. Los guardas de seguridad escoltan a una sección por turno a las clases artísticas impartidas en las nuevas instalaciones del CEAJOVEN, dicha sesión tiene una duración aproximada de 2 horas, por lo que generalmente se convive con dos secciones por día de taller. Las sesiones artísticas inician con un calentamiento y se desarrolla la preparación coreográfica y física de los muchachos participantes. Deben aprender, practicar y memorizar nuevos pasos en la mayoría de sesiones; así como deben practicar un resultado grupal positivo: manejo de coordinación, espacio personal y grupal, espera de turnos, dominio de la coreografía, enfrentarse al miedo escénico y a bailar con público. Como cierre a este proceso, los muchachos bailaron en dos actividades importantes durante el desarrollo del Taller: En la Presentación Folclórica Cárcel San Sebastián y en La Feria Penitenciaria en la Antigua Aduana.

Por otro lado, la sección D1 y la de Máxima Seguridad trabajaron en el área de las Artes Plásticas: Desarrollaron manualidades, tarjetas navideñas para su familia, dibujos en

\section{psteo-


distintas técnicas, construcción con materiales distintos, pintura de dibujos impresos, etc. Al igual que las primeras secciones, estos jóvenes tienen programada su clase con anticipación y llegada la hora son trasladados por personal de seguridad a las aulas con las que cuenta el Centro. La sesión artísticas con la sección D1 tiene una duración de 2 horas aproximadamente y con Máxima Seguridad se trabajó hasta de 3 horas seguidas. Como finalización de sus proyectos, los jóvenes pueden dejarse sus trabajos y materiales con los que seguir trabajando, así como obsequiar a sus familiares las manualidades hechas para ellos.

Algunas de las temáticas tratadas explícita o implícitamente en las sesiones son las siguientes:

Manejo de la expresión corporal, conocimiento del cuerpo

Trabajo de grupo

Expresión por medio de la voz, manejo y regulación de la voz

Expresión por medio del cuerpo, sin voz.

Expresión por medio de la pintura: abstracta o directamente

Valor, peso y uso de las palabras (poesía)

Enfrentarse al público, saberse observado, estar en escena

Manejo de "los nervios", canalización de la presión

Compromiso con otros, dependo y dependen de mí.

Confianza en los otros y en mí mismo para llevar a cabo la tarea (el baile, la canción, la obra de teatro). Cuándo delegar y cuándo asumir

No existe la vergüenza: Expresar por medio del arte no admite juicios 
Validación de mis ideas y respeto a la variedad de las de mis compañeros

\section{Conclusiones}

Los 33 jóvenes que conforman las secciones $\mathrm{C} 1, \mathrm{C} 2, \mathrm{~B} 2$ y $\mathrm{E}$ inician en una experiencia artística en el área de la Danza cuando la trabajadora social María Fernanda Mora Calvo les invita a formar parte del grupo coreográfico Danza Libre. Es nuevo para la mayoría de ellos el trabajo corporal, la expresión corporal, el entrenamiento muscular y la disciplina que se requiere para ver resultados. Se involucran aspectos incluso de alimentación, de ejercicios diarios recomendados a cada uno de ellos para mejorar su condición física y la solicitud imprescindible de no consumir drogas los días de ensayo que los muchachos cumplen.

El internarse en el mundo coreográfico y aceptar las disposiciones y los requisitos para mantenerse entrenando hace que los jóvenes no sólo dediquen un par de horas dos veces a la semana al ensayo de las coreografías. El tiempo dedicado a formar parte de un grupo coreográfico se intensifica en relación al nivel de compromiso que se tenga con este. La disciplina y la entrega van más allá de esas cuatro horas semanales, también abarca cada hora de acondicionamiento físico que, individualmente o por celdas, estos muchachos realizan, cada decisión respectiva a su alimentación y a su consumo de drogas tanto los días de ensayo como los días de entrenamiento físico.

Uno de los objetivos planteados en el taller fue: "Desarrollar en los participantes respeto y validación hacia sus propias ideas y hacia las de los demás a pesar de las

\section{psico


diferencias". Este objetivo se cumple cuando se analiza el trabajo en equipo. Se consiste precisamente en probar a los participantes en su propio desempeño. Por ejemplo, cuando no entienden un paso y piden ayuda, cuando se les asigna líderes para que les guíen en la rutina de baile, cuando se les pide que incluyan sus ideas para alguna parte de la coreografía y se les presenta la oportunidad de crear, de desarrollar talentos que, sin estas oportunidades, seguirían desconocidos para ellos mismos y además para que aprendan a validar y respetar las ideas de los otros.

De ese apoyo interpersonal depende en gran parte los resultados de una propuesta artística como esta: $\mathrm{Si}$ los muchachos se burlan de las ideas expuestas tanto por sus compañeros como por los que fungen como dirigentes profesionales, y los líderes de grupo ridiculizan las diferentes propuestas, entonces no hay resultados positivos. El grupo tiende a seguir a un líder en cada una de las secciones y si este no ve la participación coreográfica como aceptada y “cool”, entonces los demás no participarán aunque lo encuentren atractivo.

El éxito de las propuestas artísticas se basa en la identificación que sienta la población con lo que se le está planteando. Se vio ejemplificado con la coreografía en el ritmo de Folclore (baile típico), en la que únicamente 3 muchachos participaron porque no es lo que está de moda ni es lo más aceptado por el resto del grupo. De estas tres participaciones, sólo un joven lo hizo porque realmente quería bailar, los demás lo hicieron más por salir de la cárcel o por contratos de comportamiento con la trabajadora social.

Cuando se trata de definir capacidades de logro y de cumplimiento de metas por medio de la realización y finalización de proyectos artísticos, el final de la coreografía y los 
aplausos del público, tanto en San Sebastián como en la Feria Penitenciaria trae a los jóvenes el sabor del logro, de haber cumplido, de haber finalizado un proyecto, de haber hecho las cosas bien. Y para personas con tan poca credibilidad en sí mismos, reincidentes de los diferentes crímenes que han cometido, que salen y vuelven a entrar a prisión desde su adolescencia, este es un sabor desconocido del que inmediatamente quieren más. De eso se trata, de atraerles hacia el sentimiento de autoafirmación, hacia el lugar donde se vale darse crédito, felicitarse, sentirse satisfecho sin que exista ningún reproche ni ninguna culpa.

Los tres grupos diferentes terminan el proyecto queriendo iniciar otro y se ven animados por factores externos como la familia en las distintas ocasiones: en el caso del grupo Danza Libre, por medio de la presentación en la antigua Aduana donde las familias los acompañaron y aplaudieron; en el caso de la sección D1, el tema de la familia fue siempre relevante e influenciaba las temáticas de elección y la elaboración de trabajos, y finalmente con el privado de libertad en Máxima Seguridad que materializó su aprecio por medio de un regalo hecho manualmente a su familia.

Todos los días hay noticias acerca de crímenes, del hacinamiento en las cárceles, de los ajustes de cuentas, del tráfico de drogas, de cómo aumentan las muertes por sobre dosis de alguna de ellas, etc. Sin embargo, es una realidad escrita en un periódico o detrás de un televisor. Presenciarla en vivo y a todo color genera un golpe de conciencia acerca de la sociedad en la que se vive e inevitablemente un cuestionamiento acerca de si es realmente fructuoso el método propuesto por el sistema penitenciario nacional._Estas interrogantes generan crecimiento y madurez y llevan a querer seguir investigando acerca de las maneras 
en que se plantean las soluciones a la delincuencia y a la falta de oportunidades de educación en el país. El tiempo invertido con estos jóvenes produce la urgencia de continuar colaborando con el desarrollo emocional-psicológico de ellos, procurándoles alternativas para pasar el tiempo y que la duración de su estadía en la cárcel tenga un propósito, un resultado formativo positivo.

Queda claro además que la decisión que los muchachos toman al querer participar de estas oportunidades formativas, es una decisión personal y guiada por diferentes motivaciones. La historia de vida de cada uno de ellos puede determinar que quieran o no hacer de su tiempo en la cárcel algo productivo: terminar la escuela, el colegio, sacar un oficio. Por lo tanto la Psicología se vuelve una rama indispensable de acompañamiento en este proceso para intentar instrumentar a la persona a tomar las mejores alternativas de inversión de tiempo en condena y a motivarles a alejarse del negocio de las drogas, el sicariato y otros que se manejan dentro de la cárcel. Que los privados de libertad tengan la oportunidad de elegir se basa en cuántas opciones se les ofrezca y definitivamente el arte debe formar parte de este grupo de posibilidades que les instruyan, les guíen y les provean de herramientas tanto para invertir su tiempo y energía como para expresar sus vivencias y emociones. 


\section{Referencias}

Defensa de Niñas y Niños Internacional, Compendio de instrumentos nacionales $e$ internacionales, Justicia Penal Juvenil, Ed. DNI sección Costa Rica, 2006.

Mora, A.L., \& Gámez, I. (2001). En busca de oportunidades para adolescentes y jóvenes infractores: una propuesta de intervención. UNICEF, Costa Rica.

Obando, M. (2008). Atención Profesional en el Sistema Penitenciario nacional, lineamientos técnico-operativos. Decreto 33876-J, 2008. 